\title{
Apoyo educativo y de pares en las disciplinas en ciencia, tecnología, informática y matemáticas
}

\section{Educational and peer support in the disciplines of Science, Technology, Information Technology and Mathematics}

JIMÉNEZ-LEÓN, Rodolfo†*, MAGAÑA-MEDINA, Deneb Elí, CISNEROS-COHERNOUR, Edith y AQUINO-ZÚÑIGA, Silvia Patricia

Universidad Juárez Autónoma de Tabasco, División Académica de Ciencias Económico Administrativa. Av Universidad s/n, Magisterial, 86040 Villahermosa, Tab.

ID $1^{\text {er }}$ Autor: Rodolfo, Jiménez-León / ORC ID: 0000-0003-2635-9479, CVU CONACYT ID: 567386

ID $1^{\text {er }}$ Coautor: Deneb Elí, Magaña-Medina / ORC ID: 0000-0002-8579-596X, Researcher ID: 1-5521-2017, CVU CONACYT ID: 253765

ID $2^{\text {do }}$ Coautor: Edith, Cisneros-Cohernour / ORC ID: 0000-0003-2319-1519

ID $3^{\text {er }}$ Coautor: Silvia Patricia, Aquino-Zúñiga / ORC ID: 0000-0002-7223-8582

DOI: $10.35429 / J O T E .2019 .9 .3 .27 .34$

Recibido 15 de Julio, 2019; Aceptado 25 de Septiembre, 2019

\begin{abstract}
Resumen
Objetivos: Pocos son los estudios, los cuales identifican el apoyo que reciben los estudiantes de educación media superior en la toma de decisión por las carreras en ciencia, tecnología, ingeniería y matemática. El objetivo del presente estudio consiste en conocer las narrativas de los estudiantes de acuerdo a sus interacciones dentro de su entorno social y escolar, comprendiendo el interés individual del estudiante en términos de dominio, gustos, participación, curiosidad y apego a las áreas. Metodología: Se realizó una sesión de grupo focal con estudiantes de la carrera de matemáticas. A partir de tres preguntas detonantes que valoraron un total de 10 dimensiones en el grupo, que permitieron ajustar y enriquecer la estructura teórica mediante el método analítico sintético para descubrir relaciones y características generales. Contribución: Las áreas de interacción entre amigos dentro de los roles sociales y la tutoría por parte de los profesores en el contexto escolar, son actividades que fortalecieron la toma de decisión para las personas que participaron en el grupo focal. Se definen dimensiones concretas para la intervención en beneficio de la inclusión social en comunidades en desventaja dentro de las disciplinas CTIM debiendo ser tomadas en cuenta, especialmente por especialistas en estas áreas.
\end{abstract}

Apoyo educativo, Interés, Elección de carrera

\begin{abstract}
Objectives: There are few studies, which identify the support received by high school students in the decision making process for careers in science, technology, engineering and mathematics. The objective of the present study is to know the narratives of the students according to their interactions within their social and school environment, understanding the individual interest of the student in terms of mastery, tastes, participation, curiosity and attachment to the areas. Methodology: A focus group session was held with students of the mathematics career. From three trigger questions that assessed a total of 10 dimensions in the group, which allowed adjusting and enriching the theoretical structure through the synthetic analytical method to discover relationships and general characteristics. Contribution: The areas of interaction between friends within social roles and tutoring by teachers in the school context, are activities that strengthened decision making for people who participated in the focus group. Specific dimensions are defined for the intervention for the benefit of social inclusion in disadvantaged communities within the disciplines C.T.I.M. should be taken into account, especially by specialists in these areas.
\end{abstract}

Educational support, Interest, Career choice

Citación: JIMÉNEZ-LEÓN, Rodolfo, MAGAÑA-MEDINA, Deneb Elí, CISNEROS-COHERNOUR, Edith y AQUINOZÚÑ̃IGA, Silvia Patricia. Apoyo educativo y de pares en las disciplinas en ciencia, tecnología, informática y matemáticas. Revista de Educación Técnica. 2019. 3-9: 25-32.

\footnotetext{
*Correspondencia al Autor (Correo Electrónico: rdojle@gmail.com)

$\dagger$ Investigador contribuyendo como primer autor.
} 


\section{Introducción}

Las disciplinas de Ciencia, Tecnología, Ingeniería y Matematicas (CTIM) a nivel universitario son claves para la competencia internacional en el desarrollo del capital humano, este movimiento a 29 años de su creación en educación, a evolucionado en cinco grandes momentos : 1) Agenda política de CTIM; 2) CTIM promulgada como CTIM; 3) CTIM interpretado como C.T.i.M.; 4) El auge de la educación CTIM integrada (Blackley y Howell, 2017). Siendo Sanders (2009) quien definió la "educación CTIM integrada" como enseñanza y aprendizaje entre dos o más materias CTIM o entre una materia CTIM y una materia no CTIM tales como las artes. También describió un enfoque pedagógico de "diseño intencional e investigación” (Sanders, 2009, p.21). Se apertura un nuevo momento para las investigaciones en CTIM, explorar los repertorios interpretativos de los estudiantes para narrar su identidad en transición a la universidad y cómo éstos pueden persistir en las disciplinas; así como la experiencia escolar y el apoyo de los padres los cuales juegan un papel vital en la comprensión del desarrollo profesional de CTIM en la sociedad (Li, Mau, Chen, Lin, y Lin, T., 2019).

Hazari, Sonnert, Sadler y Shanahan, (2010) y Kanter (2009) han demostrado que los ingredientes clave en el interés de los estudiantes en las carreras C.T.I.M. son la oportunidad de participar en experiencias relevantes, reales, del mundo real. Además de las habilidades y el interés, los estudiantes que aspiran a carreras C.T.I.M. deben prepararse para ello. La motivación intrínseca y el interés conduce a una mayor persistencia cuando los estudiantes participan en tareas desafiantes (Hidi y Renniger, 2006).

Para Martínez y Camarena (2015) los sistemas de educación deben ser sensibles a los avances de la ciencia y la tecnología, en términos de ajustar constantemente los modelos de formación y promoción de la educación de los individuos. En esta perspectiva los estudiantes en su formación académica deben construir y desarrollar conocimiento, estrategias y habilidades necesarias que les permitan participar en los procesos de formulación e identificación de problemas en la cuarta transformación tecnológica 4.0 (Buisán y Valdes, 2017).

\section{Estudios previos}

Se suele considerar que las matemáticas son una materia menos popular (Bragg 2007), y la actitud negativa de los estudiantes hacia las matemáticas puede crecer con la edad (Utsumi y Mendes 2000). Sin embargo, los estudiantes mencionaron que las matemáticas siguen siendo ventajosas en algunas maneras. Primero, los estudiantes en el estudio de Hillel y Perrett (2006) determinaron el concepto de las matemáticas desde una perspectiva micro para ser un énfasis en el cálculo de números, y desde una perspectiva macro a ser un sistema para la vida cotidiana y una forma de pensar.

Las matemáticas han sido reconocidas como cruciales para el acceso y el éxito de los estudiantes en el campo de Ciencia, Tecnología, Informática y Matemáticas, debido a su alta exigencia matemáticamente; la cual proporciona al ciudadano habilidades de empoderamiento para la vida privada y social (Hernandez-Martinez, Black, Williams, Davis, Pampaka y Wake, 2008).

Los estudiantes de matemáticas se caracterizan en la problematización y el cuestionamiento de tareas y situaciones, fundamentales para la resolución de los problemas a través del uso de diversas representaciones, las cuales generan habilidades para la interpretación y comunicación de los resultados. Características internas para la disposición favorable hacia el estudio de esta disciplina (Martínez y Camarena, 2015).

Li, Mau, Chen, Lin, y Lin, (2019) a identificado a las preguntas por dimensiones: Interés personal, genero, etnicidad, apoyo del profesor, influencias familiares, sueño infantil, puestos de trabajo, rendimiento académico, CTIM expectativas de resultados de carrera, tendencias del mercado laboral, trabajos bien pagados, ambientes escolares, autoeficacia matemáticas, educación CTIM. Usher (2009) ha adaptado instrumentos para estudiantes de secundaria, de acuerdo a Zeldin y Pajares (2000) esto permite obtener información de eficacia en la que se cree subyacen las creencias de autoeficacia de los estudiantes en matemáticas. 
En México la línea de investigación por el interés por las carreras en Ciencia, Tecnología, Ingeniería y Matemáticas apenas inicia (Avedaño y Magaña, 2018). Conocer los instrumentos de los investigadores que interpretan las narrativas de los estudiantes por el interés y la motivación en las carreras CTIM, es una necesidad actual en el mundo científico (Clark y Thompson, 2016).

\section{Descripción del método}

Para Schmidt y Kelter (2017) generar un grupo focal requiere del consentimiento de los participantes, en este estudio se generaron formularios de autorización de participación y asentamiento de las narrativas de los estudiantes, con el objetivo de permitir grabar sus narraciones y se optó por el acceso libre a los estudiantes en caso de irse si lo desean.

De acuerdo con el estudio de Schmidt y Kelter (2017) se establecieron 5 etapas para el desarrollo del grupo focal: 1) Bienvenida, 2) Presentación de los participantes, 3) Reglas de operación, 4) Desarrollo y 5) Conclusión.

Por lo que se detonaron solo tres preguntas: ¿Qué te motivo al estudiar una carrera en ciencias, tecnología, informática y matemáticas?; ¿Qué factores contribuyeron para que tú entraras? y ¿Qué nos recomendarías para que otros ingresen a estas disciplinas? Las cuales permitieron la amplitud de la investigación. Las entrevistas fueron grabadas digitalmente y fueron transcritas por el autor. Como Merriam (1998) señaló, este formato "le permite al investigador responder a la situación actual, a la cosmovisión emergente del encuestado ya nuevas ideas sobre el tema" (p. 74). Por esta razón, elaboré preguntas lo suficientemente generales como para permitir a los participantes la libertad de responder en la dirección que eligieran, y utilicé las sondas para buscar más detalles, aclaraciones o ejemplos (Merriam, 1998).

\section{Participantes}

Con la ayuda de los administradores universitarios, los autores reclutaron a estudiantes que se especializan en una facultad de matemáticas de un instituto de educación superior en el sureste mexicano, específicamente en el estado de Yucatán. Enfocándose en estudiantes de primer y último año que habían alcanzado un promedio aprobatorio académico.

ISSN-2523-2460

ECORFAN $^{\circledR}$ Todos los derechos reservados
Los autores se enfocaron en los estudiantes de clase superior porque estarían más informados sobre los factores que promueven su éxito en C.T.I.M. Es importante tener en cuenta que este es un estudio en curso. Hasta el momento, se reclutaron 11 estudiantes cuyas experiencias se compartirán en este estudio actual. La tabla 1 proporciona detalles en rango de edad, tipo de carrera dentro de la facultad de matemáticas y el promedio actual de los participantes. Los seudónimos fueron optativos por parte de los participantes.

\begin{tabular}{|c|c|c|c|c|}
\hline \multicolumn{5}{|c|}{ Descripción de la muestra } \\
\hline $\begin{array}{c}\text { Nombre o } \\
\text { Seudónimo }\end{array}$ & Edad & Carrera & Facultad & Promedio \\
\hline Dreidy & 20 & $\begin{array}{l}\text { Licenciatura } \\
\text { en } \\
\text { Matemáticas }\end{array}$ & Matemáticas & 73.5 \\
\hline Andrea & 20 & \begin{tabular}{lr}
\multicolumn{2}{l}{ Licenciatura } \\
en Ciencias \\
de \\
Computación
\end{tabular} & & 87.5 \\
\hline Alan & 22 & $\begin{array}{l}\text { Ingeniería en } \\
\text { computación }\end{array}$ & & 88 \\
\hline Mario & 25 & $\begin{array}{l}\text { Ingeniería en } \\
\text { computación }\end{array}$ & & 81 \\
\hline Sergio & 23 & $\begin{array}{l}\text { Ingeniería de } \\
\text { software }\end{array}$ & & 76 \\
\hline Armando & 24 & \begin{tabular}{lr}
\multicolumn{2}{l}{ Licenciatura } \\
en Ciencias \\
de r la \\
Computación \\
\end{tabular} & & 78 \\
\hline Álvaro & 23 & \begin{tabular}{lr}
\multicolumn{2}{l}{ Licenciatura } \\
en Ciencias \\
de r la \\
Computación
\end{tabular} & & 70 \\
\hline Alonso & 21 & \begin{tabular}{lr}
\multicolumn{2}{l}{ Licenciatura } \\
en & Ciencias \\
de & la \\
\multicolumn{2}{c}{ Computación }
\end{tabular} & & 88 \\
\hline Alan & 20 & $\begin{array}{l}\text { Licenciatura } \\
\text { en ciencias de } \\
\text { la } \\
\text { computación }\end{array}$ & & 85 \\
\hline Luis & 19 & $\begin{array}{l}\text { Licenciatura } \\
\text { en } \\
\text { Matemáticas }\end{array}$ & & 91 \\
\hline Omar & 19 & $\begin{array}{l}\text { Licenciatura } \\
\text { en } \\
\text { Matemáticas }\end{array}$ & & 95 \\
\hline
\end{tabular}

Tabla 1 Descripción de la muestra

Se identifica solo a dos mujeres dentro de la muestra. Sus especialidades iban desde las matemáticas hasta la computación. La muestra estaba compuesta por estudiantes de excelencia y media competencia académica. Específicamente, el estudio incluyó tres ciencias de campos de estudios (Ingeniería, Matemáticas y Tecnología). 


\section{Recopilación de datos}

Se realizó un grupo focal, en profundidad, que duró aproximadamente una hora. Antes de comenzar el grupo focal, los participantes firmaron un formulario de consentimiento y al finalizar la sesión un documento de asentamiento informado (Schmidt y Kelter, 2017); Durante la sesión, se les preguntó sobre sus motivaciones en el estudio por las disciplina; factores que contribuyeron en las experiencias académicas y sociales al ingreso a la facultad de matemáticas y sus recomendaciones para el ingreso de otros estudiantes por las disciplinas C.T.I.M.

Los autores registraron observaciones sobre las formas en que los participantes respondieron a las preguntas y su disposición a participar en el grupo focal. También se realizaron entrevistas telefónicas de seguimiento con participantes para solicitar datos demográficos. Todas las respuestas fueron grabadas en audio $y$ transcritas textualmente.

\section{Análisis de datos}

Los autores utilizaron análisis comparativos constantes en notas de investigación, observaciones y transcripciones durante las etapas de desarrollo del grupo focal para identificar temas recurrentes o únicos. Específicamente, a medida que los datos se recopilaron y transcribieron, los autores consultaron estudios de investigación y tomaron notas auto-reflexivas para contribuir a los estudios cualitativos (Clark y Thompson, 2016). Los datos cualitativos del grupo focal, se organizaron, administraron y decodificaron a través de un mapa de ubicación de conceptos clasificando las palabras de acuerdo a las dimensiones teóricas revisadas.

Los autores utilizaron la codificación abierta, que implicaba analizar los datos línea por línea, para identificar los temas. La codificación línea por línea permitió que los temas emergieran de los datos y se agregaran en patrones de respuesta. Al analizar los hallazgos, se presentan extractos de las respuestas de los participantes textualmente para preservar la esencia de las voces de los participantes.
Credibilidad y confiabilidad. Se emplearon técnicas para conservar la integridad y preservación de la autenticidad de las voces de los participantes con la experiencia en métodos cualitativos (Cisneros, 2019; Martínez, 2014 y User 2004), Durante la etapa de cierre los participantes recibieron sus transcripciones como grupo para asentar la credibilidad de las narraciones y realizar ajustes en sus declaraciones.

Limitaciones. De acuerdo al calendario escolar, el grupo focal se desarrolló durante finales del semestre por lo cual la afluencia de estudiantes dentro de la institución, generó una disminución en la selección de la muestra. Por lo tanto identificamos a los promedios y las edades de los participantes como agrupación.

\section{Resultados}

En esta sección, se resumen cuatro temas principales que surgieron del grupo focal: (a) apoyo de grupos de pares, (b) participación en experiencias educativas C.T.I.M. (c) preparación en orientación vocacional (d) Identidad científica. El primer tema incluye descripciones de los participantes sobre el valor del apoyo del grupo de compañeros y la influencia positiva que tienen los compañeros en el éxito académico. El segundo tema explora la importancia de la participación en olimpiadas y ferias C.T.I.M. Además, su participación en estas actividades especializadas les ayudó a ampliar su comprensión de las profesiones y oportunidades de C.T.I.M. El tercer tema trata sobre la identificación del nivel bajo de preparación en las áreas de orientación vocacional y profesional del sistema medio superior. El cuarto tema describe la percepción de la identidad científica que cuentan los estudiantes de las disciplinas C.T.I.M; se delinean los temas y se presentan citas de los participantes para preservar el aspectos esenciales de su experiencia.

\section{Apoyo de grupos y pares}

Los participantes en discusión grupal identificaron dos tipos de apoyos de grupos y pares para la decisión de la carrera estos se describieron de diferentes maneras: (1) Sirvió como aclaración de información y diferenciación de disciplinas para su toma de decisión de carrera; (2) Se consulta la aprobación para la obtención del soporte. 
Por ejemplo Alan, un alumno de la licenciatura en ciencias de la computación cuyo interés en la etapa infantil se encontraba en la tecnología, esta se incrementó a través de los años.

Llegado el momento de elección de carrera recurrió al apoyo de pares a través de un egresado quien le informo las diferencias de los campos de acción de las carreras; específicamente, el compartió lo siguiente:

Participante 3. "Desde pequeño igual sabía que quería computación; solo que no sabía exactamente que carreras, de hecho hasta mi último año de prepa cuando entre, fue cuando me puse a investigar sobre las carreras que habían de computación acá en Yucatán, y pues eh, con un amigo mayor igual que había estudiado LCC, ese que me empezó a explicar las diferencias de las carreras, y luego yo me puse a investigar los planes de estudio y todo; y fue la que más, Ciencias de la computación fue la que más me termino llamado".

Todos los estudiantes hablaron sobre la búsqueda de información y la identificación de apoyo a través de pares con experiencia para la aclaración de dudas, ya que todos los participantes mencionaron sobre la tensión que vivieron al elegir una disciplina C.T.I.M. al no encontrar la carrera específica por motivos geográficos, económicos y oferta educativa, durante la toma de decisión. En esta sección podemos observar el momento en el que Mario notó:

Participante 1. "En un principio estaba como un poco inseguro, porque era una cuestión, que era ingeniera, todos tienen la consigna que va estar matado, va estar difícil, puras matemáticas y física y todo eso, pero yo tenía más ganas de ir por lo que yo estaba buscando en un principio, costara lo que tuviera que costar...".

Cuando un estudiante finalmente toma una decisión vocacional, es porque la opción elegida se ha considerado la más aceptable al considerar otras opciones. Aquí inicia un proceso de creación de estrategias por parte del estudiante, utilizado en el proceso de circunscripción; Mario compartió su proceso:
Participante 1: "Yo quería estudiar algo relacionado con animación digital o modelado $3 \mathrm{~d}$ entonces las opciones que yo había visto..., pero de precio eran muy elevadas, entonces a mí me dijeron pues que no, que buscara otras, que se pareciera, porque a mi igual me gustaba mucho el área de informática...entonces cheque una alternativa a esa misma carera y luego salió esta, que era aquí en la UADY, que era ingeniería en computación y que entre su sección de optativas estaba lo que yo quería estudiar en un principio como carrera entera, estaba como optativa acá, entonces a mí me pareció una buena idea entrar en ese terreno".

Los estudiantes que abandonaron sus preferencias iniciales por alternativas más alcanzables de acuerdo a los factores geográficos, económicos y académicos deseables presentaron un compromiso. Estas se ubicaron al seleccionar los mapas curriculares de las diferentes licenciaturas para la elección de carrera. Andrea quien nos platicó su experiencia y la determinación de su elección:

Participante femenina 9: “...en la prepa estuve tuve la duda, fue mi etapa de duda, porque estaba entre por escoger carreras relacionadas a literatura...pero por ejemplo en la UADY, la que dan es literatura mexicana y esas a mí no me gusta, entonces he, y retome lo de la computación porque siempre, he, era la más apegada a las cosas de computación en la familia, y entonces dije: -a, voy a intentarlo...Por ejemplo primero descarte la de ingeniería en computación porque es más dedicada a hardware, y estaba entre ingeniería en software y la de ciencias de la computación, que es la que finalmente elegí'.

Andrea no solo consulto la aprobación de su hermana mayor, quien había estudiado anteriormente la carrera de ciencias de la computación; sus padres tenían una consigna al someter su elección a la institución de educación superior por la calidad educativa, reconocimiento y accesibilidad que la hermana había experimentado en su licenciatura. Andrea genera un compromiso de manera familiar y una aprobación obteniendo el soporte en su toma de elección. 


\section{Participación en experiencias educativas}

Dentro de los factores que propiciaron el acercamiento a las disciplinas C.T.I.M. en el nivel medio superior por parte de los participantes se ubicaron dos actividades promovidas por los sistemas educativos; Las ferias y las olimpiadas académicas, espacios de fortalecimiento de habilidades y competencias para los estudiantes. En dichas experiencias académicas se observan esfuerzos constructivos y creativos para la solución de problemas; también identificando términos de dominio, gustos, participación, interés, curiosidad y aprecio por la disciplinas C.T.I.M. Así lo demostró Omar, estudiante con un alto nivel en Matemáticas:

Participante 7: "Pues al menos en secundaria y en la primaria, me llevaron también para concursar a la olimpiadas de las matemáticas, y pues ya cuando llegue a la fase "choncha" poderosa, donde te podían demostrar algo, desde ahí me di cuenta, pues me gustaría poder hacer esto, porque me gustan las matemáticas, y pues me entere pues que en la UADY, hay licenciatura en matemáticas, en contrario con quien alguien dijo que no quería profundizar tanto en las matemáticas, a mí me interesa profundizar más en matemáticas".

El nivel de interés de los estudiantes del grupo focal se identifican tres aspectos de influencia: a) Atención en la curiosidad y aprecio a la disciplina en el nivel básico y medio superior educativo; b) Objetivos específicos que atiende la experiencia educativa a través de la participación de los estudiantes para promover el interés en las ciencia y la tecnología; c) Nivel de aprendizaje de los estudiantes a través de la solución de problemas con características de gustos y dominios.

Podemos observar detenidamente que un factor que propicia la participación de los estudiantes en estas actividades son los profesores; en esta línea los estudiantes identificaron los apoyos recibidos durante estas experiencias. Así lo explica Luis:
Participante 10: “...Factores, pues tendría que ser mucho la parte de los concursos ¿no?, desde pequeño empecé, aja, pus era bueno en las matemáticas, pues... luego, siempre me, era como muy fastidiosos para los profesores, -¿no?-; porque solía preguntarles: ¿Quien decía, que tal cosa era así?-; -¿Por qué esta forma es así?-; -¿Por qué esto es así?-. Y usualmente te la barajean, -¿no?-; - ¡Es así!-; ¡Úsalo!-, ¡Cosas así!-, Entonces en ese sentido, pues quería saber ese tipo de cosas...".

Se identifica una necesidad por parte de los estudiantes, el sentido de apoyo a la vocación en ciencia y tecnología, promoviendo una valoración en la educación científica, que tendrá una identificación positiva o negativa, personal y social de los estudiantes en el momento de la toma de decisión.

\section{Preparación en orientación vocacional}

Los estudiantes identificaron a las materias de orientación vocacional y profesional desarrolladas en el nivel medio superior de acuerdo a sus experiencias, en un nivel bajo de aprovechamiento y expectativas por la toma de decisiones, debido a que los resultados obtenidos en las pruebas test y cuestionarios, no contribuyeron a sus intereses personales en la incursión por las carreras científicas. Así lo expreso Alan:

Participante 1: "y existe lo que es orientación vocacional y orientación profesional, pero ninguna te sugiere algo como despertar ese, -¿cómo se llama?-, interés, sobre alguna de las carreras, o que te pregunte, como estaba mencionando Mario, Hay el tipo orientación vocacional -iqué es lo que te interesa?-; y pues muchos en esa etapa de tu vida, cuando te hicieron la pregunta, muchos ¡No sé!-, entonces agarran y solo dibujan cosas, algo que les guste, y eso entregábamos. En orientación profesional fue un examen, cada una de las sesiones que habían en la semana era un examen diferente, supuestamente para saber más o menos a que área se te hacia más fácil, que habilidades tenías y si y cual era más o menos el enfoque, hacía que carreras se enfocaban en esa área, pero muchos eso no quería, podían salir bien en los exámenes en ciertas áreas, pero eso no signifique que les interese sobre esas áreas, querían sobre otras, recuerdo que a una amiga le salió para ciencias sociales o algo así y es dentista". 
También observamos la valoración de juicios y estereotipos a través de los resultados de los test vocacionales y cuestionarios de elección de carrera por parte la sociedad, no teniendo en cuenta que la propuesta de orientación y asesoramiento para América Latina se describe en base a un híbrido y el dialogo intercultural responde a las demandas de transformación de la orientación y asesoramiento. Debiendo tener especial atención a los momentos de transición del alumno en sus diferentes etapas educativas: familia-escuela, enseñanza, escuela-trabajo (McNamee,2011; Ribeiro, 2017; Perera, y McIlveen, 2018).

\section{Así lo expreso Omar:}

Participante 11:"Si, si a un amigo le salió filósofo, y enseguida le dijeron que se iba a morir de hambre".

Al cuestionar las incongruencias de los resultados en orientación vocacional y profesional. Aquí conectamos con el último punto de hallazgos en la identificación de la identidad de estudiantes CTIM.

\section{Conclusión}

El conjunto de emociones, creencias y actitudes que surgen hacia estas disciplinas son respaldadas en este grupo focal de acuerdo con Gil, Blanco y Guerrero (2006) los estudiantes se identifican con una fuerte afectividad hacia las disciplinas. De acuerdo con Sarı, Misra,y Ömer-Faruk (2018) la entrada a las disciplinas CTIM, es regulado por la actitud de los estudiantes y las percepciones de la Carrera; en el grupo focal se identificaron patrones de desarrollo de habilidades y las diferencias en la socialización (Feldon, Maher, Roksa, y Peugh, 2016), proporcionando una visión de como dentro de las experiencias en Ferias de Matemáticas, Olimpiadas y Expos vocacionales de la ciencia, la tecnología surgen patrones de ínteres, los cuales favorecen en la promoción e ingreso de nuevos estudiantes a estas disciplinas. Estos resultados surgieren que la elección de un ingreso a las carreras en CTIM está directamente influenciada por tres factores: apoyo de pares, soporte familiar y orientación educativa (Wang, 2013).
Se identifica al factor apoyo de pares como soporte para la toma de elección de las carreras en ciencia, tecnología, ingeniería y matemáticas; ya que las experiencias validan este soporte en la toma racional y consolidación de la identidad profesional de los estudiantes. Es necesario generar un replanteamiento de la orientación vocacional en la educación media superior, para satisfacer las necesidades de los estudiantes; teniendo en cuenta que debe ser un aliado y no un trámite del currículo educativo. El soporte familiar sigue siendo uno de los factores predominantes en la toma de decisión de carreras CTIM por lo que es necesario ampliar las investigaciones sobre sus consecuencias y determinaciones.

Por lo que surgen futuras preguntas de investigación: ¿Cuáles son los discursos en las experiencias de los estudiantes en relación con las disciplinas en CTIM?, ¿Cuáles son los factores sociales que apoyaron al estudiante?; Lo que nos permitira argumentar las distintas opciones del estudiante de acuerdo a las ventajas e inconvenientes para la elección de los campos en CTIM y detectar cuando el estudiante por cede ante los deseos sus padres, profesores, pares u otras presiones en la toma de decisión por las carreras en ciencia, tecnología, informática y matemáticas.

\section{Referencias}

Blackley, S. y Howell, J. (2015). A STEM Narrative: 15 Years in the Making. Australian Journal of Teacher Education, 40(7), 102-112. doi.org/10.14221/ajte.2015v40n7.8

Blackley, S., y Howell, J. (2015). A STEM narrative: 15 years in the making. Australian Journal of Teacher Education, 40(7). DOI:10.14221/ajte.2015v40n7.8.

Bragg, L. (2007). Students' conflicting attitudes towards games as a vehicle for learning mathematics: A methodological dilemma. Mathematics Education Research Journal, 19(1), 29-44. Recuperado de https://dro.deakin.edu.au/eserv/DU:30007361/b ragg-studentsconflicting-2007.pdf

Buisán, M. y Valdés, F. (2017). La industria conectada 4.0. la economía digital en España, 898(1),89-100. Recuperado de htpp:///C:/Users/alumno/Downloads/1963-

Texto\%20del\%20art\%C3\%ADculo-1962-1-1020180711\%20(1).pdf

JIMÉNEZ-LEÓN, Rodolfo, MAGAÑA-MEDINA, Deneb Elí, CISNEROS-COHERNOUR, Edith y AQUINO-ZÚÑIGA, Silvia Patricia. Apoyo educativo y de pares en las disciplinas en ciencia, tecnología, informática y matemáticas. Revista de Educación Técnica. 2019 
Clark, A. y Thompson, D. (2016). Five Tips for Writing Qualitative Research in High-Impact Journals: Moving From \#BMnoQual. International Journal of Qualitative Methods, 13.doi: $10.1177 / 16094069166441250$

Feldon, D. F., Maher, M. A., Roksa, J., y Peugh, J. (2016). Cumulative Advantage in the Skill Development of STEM Graduate Students. American Educational Research Journal, 53(1), 132-161. doi:10.3102/0002831215619942

Gil, N., Blanco, L. y Guerrero, E. (2006). El papel de la afectividad en la resolución de problemas matemáticos. Revista de educación, v. (340), 551-569.

Hazari, Z., Sonnert, G., Sadler, P. M., y Shanahan, M. (2010). Connecting high school physics experiences, outcome expectations, physics identity, and physics career choice: A gender study. Journal of Research in Science Teaching, 47(8), 978-1003. doi:10.1002/tea.20363

Hernandez-Martinez, P., Black, L., Williams, J., Davis, P., Pampaka, M., \& Wake, G. (2008). Mathematics students' aspirations for higher education: class, ethnicity, gender and interpretative repertoire styles. Research Papers in Education, 23(2), 153165.doi:10.1080/02671520802048687

Hidi, S., y Renniger, K. (2006). The four phase model of interest development. Educational Psychologist, 41(2), 111-127. doi:10.1207/s15326985ep4102_4

Hillel, J., y Perrett, G. (2006). Undergraduate students' conceptions of mathematics: An international study. International Journal of Science and Mathematics Education, 5, 439$459 . \quad$ Recuperado de http:///C:/Users/alumno/Downloads/Undergrad uate_Students_Conceptions_of_Ma.pdf

Kanter, D. E. (2009). Doing the project and learning the content: Designing project-based science curricula for meaningful understanding. Science Education, 94(3), 525-551. Recuperado de

http://citeseerx.ist.psu.edu/viewdoc/download? doi=10.1.1.686.7291\&rep=rep1\&type=pdf
Li, J., Mau, W.-C. J., Chen, S.-J., Lin, T.-C., y Lin, T.-Y. (2019). A Qualitative Exploration of STEM Career Development of High School Students in Taiwan. Journal of Career Development, 20(10) 1-15. doi:10.1177/0894845319830525

Martínez, X. y Camarena, R. (Eds.). (2005). La educación matemática en el siglo XXI. México: Instituto Politécnico Nacional.

Merriam, S. B. (Ed.). (1998). Qualitative research and case study applications in education. San Francisco: Jossey-Bass

Sanders, M. (2009). STEM, STEM Education, STEMmania. The Technology Teacher, 2(1) 20-26. Recuperado de http://vtechworks.lib.vt.edu/bitstream/handle/10 919/51616/STEMmania.pdf?sequence $=1 \&$ isAll owed=y

Sarı, U., Mısra, A. y Ömer-Faruk, Ş. (2018) The Effect of STEM Instruction on Attitude, Career Perception and Career Interest in a Problem-based Learning Environment and Student Opinions. Electronic Journal of Science Education . 22(1) 1-21. Recuperado de http://web.a.ebscohost.com/ehost/pdfviewer/pdf viewer?vid=5\&sid=1a183686-9587-430a-b4a4f8917187b687\%40sdc-v-sessmgr03

Usher, E. L. (2009). Sources of Middle School Students' Self-Efficacy in Mathematics: A Qualitative Investigation. American Educational Research Journal, 46(1), 275-314. doi:10.3102/0002831208324517

Utsumi, M., y Mendes, C. (2000). Researching the Attitudes Towards Mathematics in Basic Education. Educational Psychology, 20(2), 237-243.doi:10.1080/713663712

Wang, X. (2013). Why Students Choose STEM Majors. American Educational Research Journal, 50(5), 10811121.doi:10.3102/0002831213488622

Zeldin, A. L., y Pajares, F. (2000). Against the Odds: Self-Efficacy Beliefs of Women in Mathematical, Scientific, and Technological Careers. American Educational Research Journal, 37(1), 215246.doi:10.3102/00028312037001215 